www4.fsanet.com.br/revista

e-revist@s

Rev. FSA, Teresina, v. 18, n. 01, art. 12, p. 263-288, jan. 2021

ISSN Impresso: 1806-6356 ISSN Eletrônico: 2317-2983 http://dx.doi.org/10.12819/2020.18.01.12

\title{
Qualidade de Serviço no Ensino Superior Público: Uma Adaptação do Servperf
}

\author{
Service Quality in Public Higher Education: A Servperf Adaptation
}

Marina Benevenuto Demaria Monteiro

Engenheira de Produção pela Instituto Federal do Espírito Santo E-mail: marina.benevenuto@yahoo.com.br

Luiz Gabriel Pereira Herzog

Mestre em Engenharia de Produção pela Universidade Federal de Santa Catarina E-mail: gabriel_herzog@hotmail.com

Cintia Tavares do Carmo

Doutora em Sociologia Política pelo Instituto Federal do Espírito Santo Professora do Instituto Federal do Espírito Santo Campus Cariacica

E-mail: cintiata@gmail.com

Fernando Antônio Forcellini

Doutor em Engenharia Mecânica pela Universidade Federal de Santa Catarina Professor da Universidade Federal de Santa Catarina Campus Trindade

E-mail: fernando.forcellini@ufsc.br

Endereço: Marina Benevenuto Demaria Monteiro Rodovia Governador José Sette 184, Itacibá, CEP: 29150410, Cariacica/ES, Brasil.

Endereço: Luiz Gabriel Pereira Herzog

Campus Reitor João David Ferreira Lima Trindade, CEP: 88040900, Florianópolis/SC, Brasil.

Endereço: Cintia Tavares do Carmo

Rodovia Governador José Sette 184, Itacibá, CEP:

29150410, Cariacica/ES, Brasil.

Endereço: Fernando Antônio Forcellini

Campus Reitor João David Ferreira Lima Trindade, CEP:

88040900, Florianópolis/SC, Brasil.
Editor-Chefe: Dr. Tonny Kerley de Alencar Rodrigues

Artigo recebido em 03/11/2020. Última versão recebida em 17/11/2020. Aprovado em 18/11/2020.

Avaliado pelo sistema Triple Review: a) Desk Review pelo Editor-Chefe; e b) Double Blind Review (avaliação cega por dois avaliadores da área).

Revisão: Gramatical, Normativa e de Formatação

AGENCIA DE FOMENTOS: Conselho Nacional de Desenvolvimento Científico e Tecnológico - CNPq pelo suporte recebido para o desenvolvimento desta pesquisa. 


\title{
RESUMO
}

Este trabalho objetiva avaliar a qualidade dos serviços prestados por uma instituição de ensino superior (IES) pública por meio da adaptação do instrumento SERVPERF de Cronin \& Taylor (1992). O método da pesquisa baseou-se no desenvolvimento de uma revisão sistemática da literatura (RSL) para identificar as principais lacunas de pesquisas relacionadas ao SERVPERF em IESs e um estudo de caso aplicado em um setor de suporte ao ensino de uma instituição pública (IP) de educação tecnológica. Para isto, utilizou-se um questionário com 10 itens aplicado a 235 alunos do curso de graduação de engenharia de produção. Após validação de 192 questionários, os itens foram analisados e priorizados por meio da análise de quartis. A partir dos principais resultados classificados em prioridades crítica e alta, identificaram-se oportunidades de melhorias nos serviços pertencentes às dimensões da qualidade: tangibilidade, responsividade e confiabilidade. Salienta-se que o desenvolvimento desta pesquisa auxilia não só a proposição de melhorias referentes à avaliação da qualidade dos serviços no setor estudado mas também aos outros 21 polos universitários no estado analisado e aos diversos dispersados no Brasil.

Palavras-chave: Qualidade em Serviços. Servperf. Ensino Superior. Ensino Público.

\begin{abstract}
This work aims to evaluate the quality of services provided by a public higher education institution (HEI) by adapting the SERVPERF instrument by Cronin \& Taylor (1992). The research method was based on the development of a systematic literature review (SLR) to identify the main research gaps related to SERVPERF in HEIs and a case study applied in a teaching support sector of a public institution (PI) of technological education. For this, a questionnaire with 10 items was applied to 235 undergraduate industrial engineering students. After 192 questionnaires were validated, the items were analyzed and prioritized through quartile analysis. From the main results classified as critical and high priorities, opportunities for improvements in services belonging to the dimensions of quality were identified: tangibility, responsiveness, and reliability. It should be noted that the development of this research helps not only to propose improvements regarding the evaluation of the quality of services in the sector studied but also to the other 21 university centers in the analyzed state as well as to the several dispersed in Brazil.
\end{abstract}

Keywords: Service Quality. Servperf. Higher Education. Public Education. 


\section{INTRODUÇÃO}

O setor de serviços tem sido responsável pelo desenvolvimento do cenário econômico dos países por promover atividades que se conectam e impulsionam a sociedade (FITZSIMMONS; FITZSIMMONS, 2014). No Brasil, percebeu-se um aumento da participação deste setor na economia nas últimas décadas (IBGE, 2016, 2020). Em 2018, observou-se que os serviços representaram $76 \%$ do produto interno bruto, indicador que quantifica a produção de bens e serviços de um país em um ano (COSCIEME et al., 2020), correspondendo a um aumento de aproximadamente 1,1\% (2017-2018) (AMORIM et al., 2019; ROMANI; QUINTINO, 2019) e 1\% (2018-2019) (ALVARENGA, 2020).

O serviço é definido como uma tarefa a ser executada que apresenta valor econômico (TUKKER, 2004), com características heterogêneas (PARASURAMAN et al., 1985) e intangíveis (KOTLER, 1988), a partir do estabelecimento da relação com o consumidor (GRÖNROOS, 2004), formados pela integração de processos, habilidades pessoais e materiais (GOLDSTEIN et al., 2002). Podem ainda ser segmentados em serviços privados, quando financiados por particulares, e públicos, quando subsidiados pelo Governo (DUSSAULT, 1992). De maneira geral, os serviços públicos são caracterizados pelo atendimento das necessidades essenciais à comunidade como segurança, justiça, saúde, alimentação, transporte, moradia e educação, assegurados pela Constituição.

Dentre os serviços públicos existentes, destaca-se a educação. Considera-se este setor como um dos fatores cruciais para o crescimento da economia de um país (GAO et al., 2019). No ensino superior, especificamente, observa-se esta relação a partir do desenvolvimento do capital humano e utilização de ferramentas de tecnologia (SIRBU, 2015) por meio da capacitação dos estudantes nas universidades (OMODERO; NWANGWA, 2020) em atividades de ensino e pesquisa relacionadas ao desempenho industrial de modo a garantir a promoção do bem-estar da sociedade (GUERRERO et al., 2015).

De acordo com o Ministério da Economia (2018), apenas 30\% das instituições federais no Brasil apresentam métodos de avaliação da qualidade em seus serviços, e somente $50 \%$ dessas utilizam a opinião do usuário para a proposição de melhorias em seus processos. Dessa forma, aumenta-se a preocupação em desenvolver e aplicar métodos a partir da utilização de ferramentas quantitativas confiáveis (BROCHADO, 2009; SHANKAR et al., 2019) que identifiquem os principais problemas e, consequentemente, apresentem oportunidades de melhoria na prestação de serviços (SHURAIR; POKHAREL, 2019). 
Diversos modelos foram elaborados para avaliar a qualidade dos serviços, sendo a proposição do modelo SERVQUAL (PARASURAMAN et al., 1988) e SERVPERF (CRONIN; TAYLOR, 1992) os mais explorados na literatura. O instrumento SERVQUAL foi desenvolvido a partir do modelo de gaps (PARASURAMAN et al., 1985) com o objetivo de avaliar a qualidade de serviço prestado a partir da diferença entre o desempenho ofertado e a expectativa do consumidor por meio de cinco dimensões da qualidade (tangibilidade, confiabilidade, responsividade, segurança e empatia) distribuídos em 44 itens avaliados por meio de um questionário (PARASURAMAN et al., 1985, 1988). Em contrapartida, o instrumento SERVPERF, do acrônimo "Service Performance” (Babić-Hodović et al., 2019), foi elaborado pelos autores Cronin e Taylor (1992) a partir do confrontamento com o modelo SERVQUAL (HAQUE; SULTAN, 2019; MANDAL; GUPTA, 2019). Os autores demonstraram que a qualidade do serviço é melhor caracterizada, representando maior confiabilidade e eficácia, pela análise da percepção de desempenho advinda dos consumidores, excluindo o fator expectativa (DUTTA, 2019; YU; HYUN, 2019). Assim, a mensuração é obtida apenas pela percepção do consumidor (AKDERE et al., 2020) por meio da aplicação dos 22 itens (MOHAN et al., 2019).

Embora o SERVERF tenha sido desenvolvido há quase três décadas, ainda há pouca exploração do instrumento IESs públicas, especialmente em IP brasileiras. Portanto, o objetivo deste trabalho é avaliar a qualidade dos serviços ofertados por uma IES pública por meio da adaptação do instrumento SERVPERF, a partir da avaliação dos estudantes do curso de graduação. De maneira prática, espera-se identificar os principais serviços responsáveis pela baixa avaliação dos alunos além de propor melhorias aos serviços prestados na instituição. Ademais, o desenvolvimento deste trabalho contribui para a expansão da literatura referente à aplicação do SERVPERF em IESs devido à elaboração da RSL, até então não explorada.

Além desta seção introdutória, o presente trabalho será estruturado da seguinte maneira: RSL referente à avaliação da qualidade em IESs (seção 2); materiais e métodos utilizados para o desenvolvimento da pesquisa como a apresentação do objeto de estudo, bem como a elaboração, coleta e análise de dados do questionário baseado no modelo SERVPERF aplicado aos alunos de uma IES pública (seção 3); apresentação e discussão dos principais resultados obtidos por meio da análise de quartis (seção 4); e considerações finais, contendo as limitações de pesquisa, contribuições (teóricas e práticas) e recomendações para trabalhos futuros (seção 5). 


\section{REFERENCIAL TEÓRICO}

\subsection{SERVPERF em IESs}

O escopo desta seção está direcionado a identificar pesquisas que realizaram a avaliação da qualidade de serviços desenvolvidas em IESs, dentre eles, cursos de graduação e pós-graduação (mestrado, doutorado e pós-doutorado) por meio do instrumento SERVPERF (CRONIN; TAYLOR, 1992). Para isso, adotou-se a aplicação da metodologia da revisão sistemática de literatura (RSL). Esta metodologia promove a coleta e avaliação do conhecimento por meio da síntese teórica da literatura existente (TRANFIELD et al., 2003) a partir de uma sequência sistemática de etapas (IDRISSI; ZELLOU, 2020) e reproduzíveis (Traxler et al., 2020) para identificar e determinar tendências de pesquisas nos campos abordados (SIHAG; SANGWAN, 2020).

Utilizou-se a combinação das palavras-chaves "SERVPERF" e os sinônimos referentes às IESs de modo a garantir amplitude nos resultados que aderem aos objetivos desta RSL, resultando na query "SERVPERF" AND ("HIGH* EDUCATION" OR "UNIVERSIT*" OR "GRADUAT*" OR "INSTITUT*"), aplicadas em setembro de 2020. Definiu-se o acesso nas bases de dados Scopus (Elsevier) e Web of Science pelo fato de ambas representarem as principais plataformas eletrônicas para buscas científicas (BUSTOS \& POMARES-QUIMBAYA, 2020), responsáveis por publicações interdisciplinares e qualificadas (RASHIDI et al., 2020) em diferentes áreas de concentração, principalmente no campo da engenharia de produção (ACERBI; TAISCH, 2020). Ressalta-se a utilização do software Mendeley no gerenciamento das referências dos documentos e Microsoft Excel no auxílio ao cálculo das ferramentas estatísticas e confecção das ilustrações.

Mecanismos de buscas foram delimitados com o intuito de verificar trabalhos relacionados ao escopo desta pesquisa. Tais mecanismos são representados pelos filtros de critérios de exclusão (CE) aplicados nas bases de dados (Tabela 1). Especificamente, C1, C2, C3 e C4 estão relacionados aos filtros iniciais de triagem enquanto C5 e C6 representam critérios de elegibilidade na leitura completa dos artigos. Aqui, os critérios de exclusões foram desenvolvidos a partir do método PRISMA, responsável para auxiliar a execução de revisões sistemáticas (MOHER et al., 2009). O método tem sido desenvolvido em diversos setores, como: educação (GLODOWSKI et al., 2020), esporte (STAMBULOVA; WYLLEMAN, 2019), marketing (ZAINUDDIN; GORDON, 2020) e saúde (DEVYNCK et al., 2019; JACOB et al., 2018). Nota-se que não foram delimitados critérios de exclusões direcionados 
ao período de publicação e áreas do conhecimento para garantir confiabilidade no método. Por fim, o processo de elaboração do portfólio final de artigos selecionados é exemplificado na Figura 1.

Figura 1 - Fluxograma no método PRISMA

\begin{tabular}{clccc}
\hline CE & \multicolumn{1}{c}{ Critério } & Scopus (Elsevier) & Web of Science & Total \\
\hline CE1 & Artigos de conferências, revisões e livros & 8 & 13 & 21 \\
CE2 & Publicações em idiomas diferentes do inglês & 1 & 5 & 6 \\
CE3 & Indisponibilidade para acesso completo & 5 & 1 & 6 \\
CE4 & Duplicidade nas bases de dados & - & - & 6 \\
CE5 & Inexistência de SERVPERF em IES & 11 & 2 & 13 \\
CE6 & Incompatibilidade com o escopo da pesquisa & 1 & 5 & 6 \\
\hline \multicolumn{2}{c}{ Tabela 1 - Critérios de exclusão }
\end{tabular}

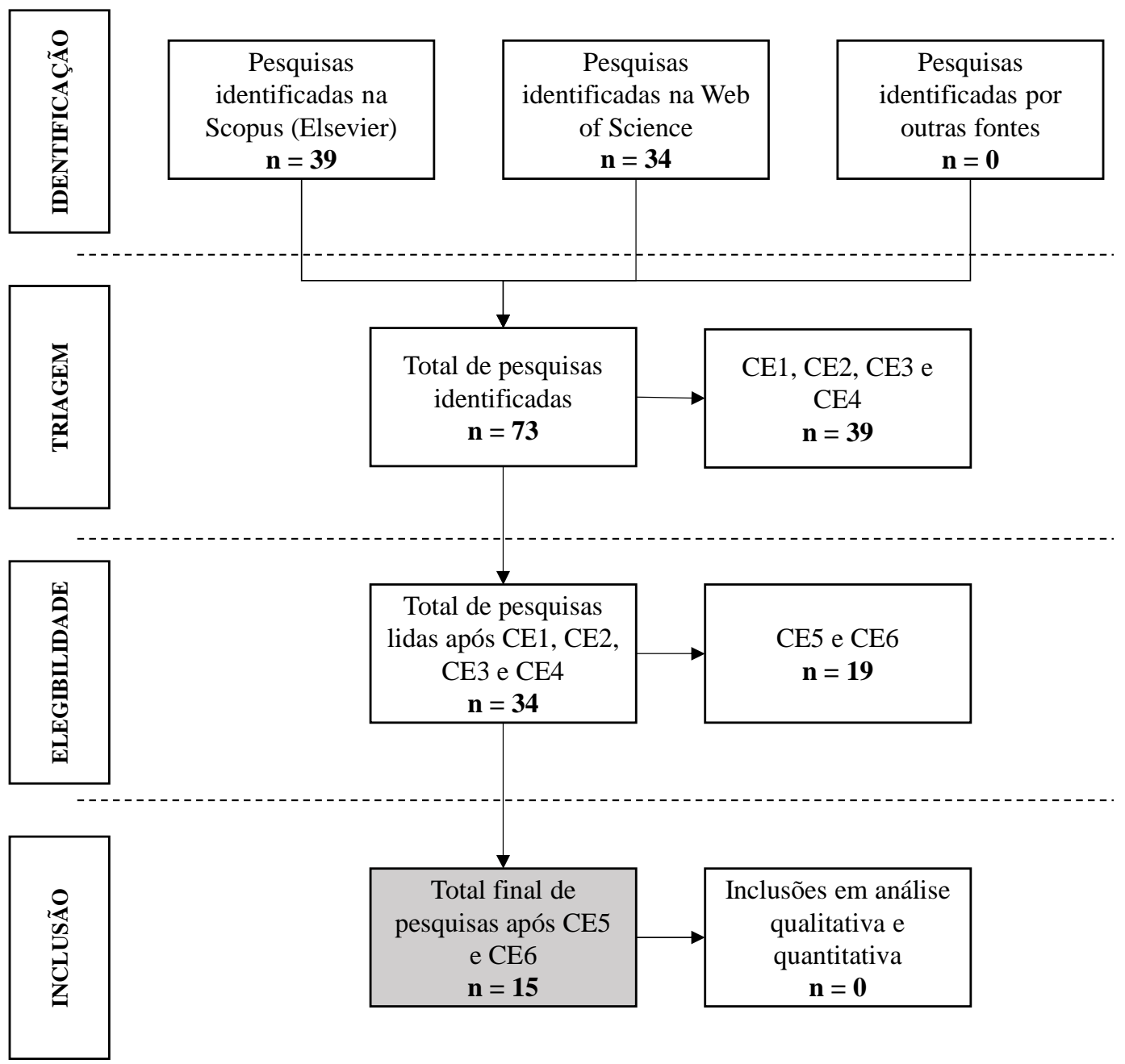

Embora o instrumento SERVPERF tenha sido elaborado há quase três décadas, encontraram-se 15 artigos aderentes ao escopo da pesquisa. Percebeu-se que países do continente asiático representaram a maior concentração de publicações (67\%), principalmente na Malásia (5), Índia (2) e Síria (2). Identificou-se que o journal Quality Assurance in 
Education (3) e Marketing Intelligence \& Planning (2) foram as principais revistas para publicações nesta área do conhecimento. Além disso, notou-se que a maioria dos trabalhos apresentaram diversidade de público-alvo para os questionários aplicados, por exemplo, apenas três trabalhos abordaram estudantes de cursos de áreas específicas (BROCHADO, 2009; MANDAL; GUPTA, 2019; RODRIGUES et al., 2011). Esta diversidade foi verificada no nível de ensino fornecido (graduação e/ou pós-graduação) e a característica da educação (público e/ou privado), exemplificado na Tabela 2.

Tabela 2 - Portfólio final. Legenda: [G] graduação e [PD] pós-graduação.

\begin{tabular}{lccccc}
\hline \multicolumn{1}{c}{ Autor (ano) } & Amostra & Curso & Nível & Característica & Região \\
\hline Mandal \& Gupta (2019) & 130 & Farmácia & PD & Privado & Índia \\
Mwiya et al. (2019) & 824 & - & G & Público & Zâmbia \\
Hamid \& Yip (2019) & 640 & - & - & Ambos & Malásia \\
Soni \& Govender (2018) & 400 & - & G & Público & África do Sul \\
Dalati \& Al Hamwi, 2016) & 107 & - & G & Privado & Síria \\
Mahmoud \& Khalifa (2015) & 259 & - & - & Ambos & Síria \\
Calvo-Porral et al. (2013) & 212 & - & G & Ambos & Espanha \\
Law (2013) & 271 & - & PD & Público & Hong Kong \\
Rodrigues et al. (2011) & 168 & Engenharia & Ambos & - & Índia \\
Brochado (2009) & 360 & Tecnologia & Ambos & - & Portugal \\
Nejati \& Nejati (2008) & 100 & - & Ambos & Pública & Irã \\
Johari \& Zainab (2007) & 274 & - & Ambos & Privado & Malásia \\
Abdullah (2006b) & 381 & - & Ambos & Ambos & Malásia \\
Abdullah 2006a) & 381 & - & Ambos & Ambos & Malásia \\
Abdullah (2005) & 381 & - & Ambos & Ambos & Malásia \\
\hline
\end{tabular}

Notou-se que diversos trabalhos utilizaram questionários testes a fim de coletar as respostas iniciais dos estudantes para reunir informações, identificar possíveis erros e desenvolver alterações necessárias de modo a permitir que o questionário final adeque-se à realidade da instituição analisada e apresente itens de fácil entendimento aos respondentes (ABDULLAH, 2005, 2006A, 2006B; BROCHADO, 2009; MAHMOUD; KHALIFA, 2015; MWIYA et al., 2019; RODRIGUES et al., 2011). Ademais, percebeu-se que 13 entre os 15 artigos utilizaram o alfa de Cronbach pois este indicador testa e valida a homogeneidade e consistência interna dos itens de avaliação de uma amostra, permitindo aumento da confiabilidade das respostas dos participantes (CRONBACH, 1951). Identificou-se a utilização de participantes pertences aos últimos anos de formação em seus respectivos cursos, de modo a permitir que os resultados sejam referentes apenas à experiência já percebida dos serviços ofertados pelas IESs. Assim, esses alunos apresentam maior 
discernimento das atividades, permitindo maior aderência à satisfação dos usuários (CALVOPORRAL et al., 2013; MANDAL; GUPTA, 2019; MWIYA et al., 2019).

Apesar do portfólio final ser representado por apenas 15 publicações, pode-se identificar três principais interações entre os autores dos trabalhos. $\mathrm{O}$ relacionamento entre as pesquisas foi permitido pela elaboração da rede de interação social por meio do NodeXL software (Figura 2) (HANSEN et al., 2011). Recentemente, a ferramenta permitiu analisar a influência da reputação dos hospitais pelos usuários a partir das postagens no Facebook (MARTIN \& GRÜB, 2020), identificar principais características presentes em manifestações políticas em redes socias (RIM et al., 2020) e, assim como neste trabalho, compreender a interação entre os autores em uma revisão de literatura (PACHECO et al., 2019). A ferramenta ilustra o direcionamento e a interação entre as pesquisas por meio de setas e círculos, respectivamente. A interação foi desenvolvida a partir da coleta manual do número de citações encontradas entre os artigos. Nota-se que trabalhos não referenciados pelos autores do portfólio final foram desconsiderados. Nesta pesquisa, os círculos maiores foram identificados por interações de no mínimo dois autores, portanto, percebeu-se que Brochado (2009), Abdullah (2006a), Abdullah (2006b) e Abdullah (2005) foram os principais autores referenciados dentre as pesquisas.

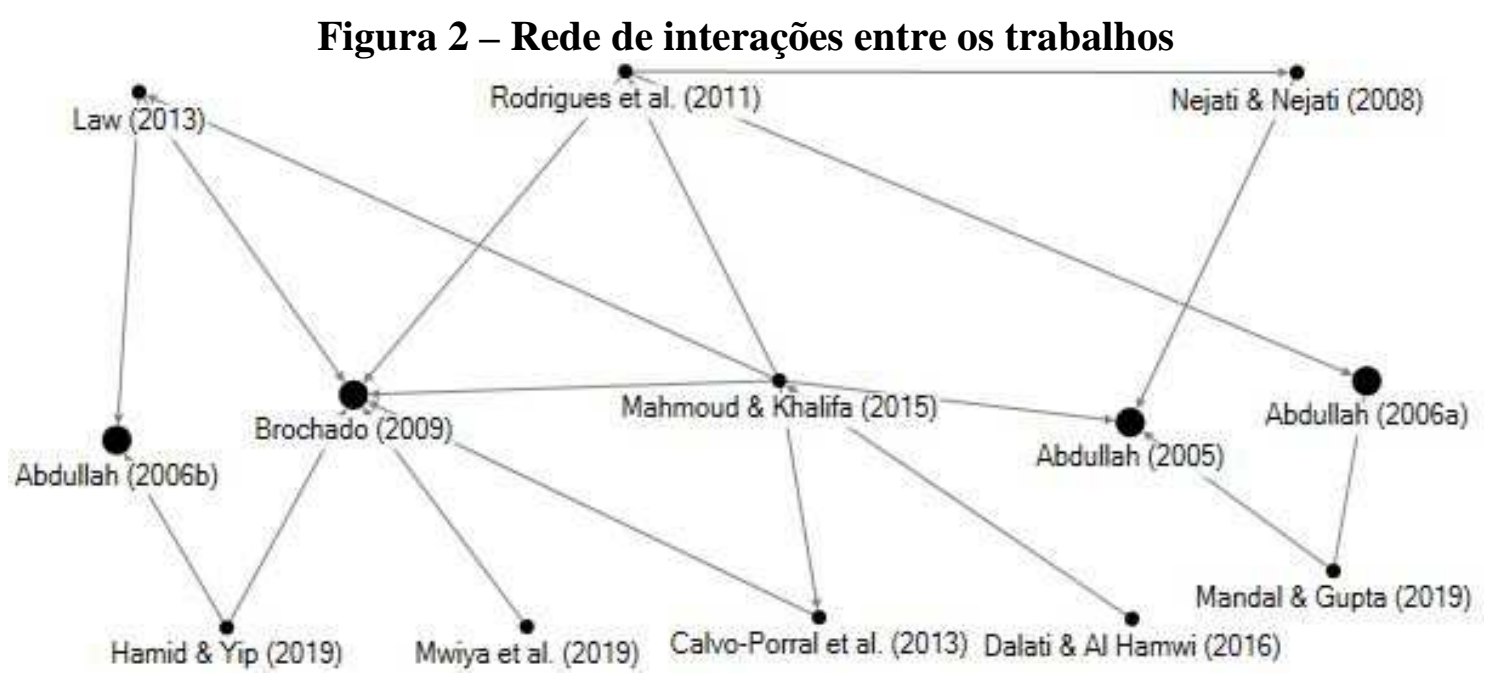

De uma maneira geral, Abdullah (2005, 2006a, 2006b) objetivaram analisar a eficiência de desempenho entre instrumentos de avaliação dos serviços em IES. Abdullah (2005) testou a escala HEdPERF para compreender os fatores determinantes na avaliação dos serviços a partir da comparação de performance com o instrumento SERVPERF enquanto 
Abdullah (2006a) e Abdullah (2006b) comparam a eficiência e o desempenho de três instrumentos (HEdPERF, SERVPERF e HEdPERF-SERVPERF). O instrumento HEdPERF foi desenvolvido com o intuito de avaliar especificamente a performance de IESs (ABDULLAH, 2006c). Os resultados das três pesquisas apontaram a preferência da utilização do questionário HEdPERF em ambientes de IESs por apresentar características que mais se adequam a esse ambiente, diferentemente de Brochado (2009) e Law (2013). Brochado (2009) examinou o desempenho dos cinco instrumentos SERVQUAL, SERVPERF, HEdPERF, importance-weighted SERVQUAL e importance-weighted SERVPERF. De acordo com os resultados, concluiu-se que os instrumentos SERVPERF e HEdPERF apresentaram melhor aderência ao escopo educacional, porém não é possível identificar o mais adequado.

Especificamente, percebeu-se que as dimensões tangibilidade e empatia apresentaram maior impacto positivo na percepção da qualidade em IESs, seja privada ou pública (CALVO-PORRAL et al., 2013) e estão diretamente relacionadas à promoção do valor nessas instituições (SONI; GOVENDER, 2018), diferentemente dos achados de Brochado (2009) em que ambas são as últimas. A tangibilidade é definida pelos aspectos físicos promovidos pela instituição, como por exemplo, aparência interna e externa, equipamentos e laboratórios enquanto a empatia é caracterizada pelo comportamento e conduta da equipe (professores e colaboradores) com os estudantes, permitindo o atendimento de suas necessidades por meio da atenção individual, como confirma Mahmoud e Khalifa (2015).

Estudantes avaliaram a tangibilidade percebida nos serviços de setores de apoio ao ensino, como as bibliotecas, como fator essencial na promoção da qualidade, incluindo a disponibilidade de acervo, seja presencial ou eletrônico. Dessa forma, deseja-se apresentar plataformas eletrônicas que possuam facilidade no acesso e eficiente interatividade com os colaboradores. Essa interatividade entre estudantes e equipe da biblioteca é compreendida pela dimensão responsividade (JOHARI; ZAINAB, 2007). Aqui, características como a disposição em ensinar (modo presencial, online e/ou telefone) a pesquisa pela disponibilidade dos documentos (JOHARI; ZAINAB, 2007) e a habilidade de atender os estudantes são consideradas cruciais na avaliação da qualidade desses serviços (MAHMOUD; KHALIFA, 2015).

Apesar da verificação de aplicação dos questionários em IESs públicas (Tabela 2), apenas três trabalhos intencionalmente investigaram a influência e as características das dimensões da qualidade presentes nessas instituições (CALVO-PORRAL et al., 2013; HAMID; YIP, 2019; MWIYA et al., 2019). Notou-se que a dimensão confiabilidade 
representa o dobro quando comparada às instituições públicas, indicando oportunidades de ações de melhorias nessa vertente (HAMID; YIP, 2019). Percebeu-se ainda que instituições privadas possuem melhores programas de avaliação da qualidade dos serviços do que centros públicos, e apresentam melhores resultados em quase todas as dimensões da qualidade, exceto tangibilidade, devido ao desenvolvimento da profissionalização dos estudantes e a sua relação com o mercado de trabalho (CALVO-PORRAL et al., 2013).

Diversos autores abordaram a necessidade de ampliação da utilização do questionário em outros segmentos, neste caso, cursos e/ou áreas não acadêmicas, como a principal limitação de pesquisa identificada. De acordo com os resultados, as generalizações sobre os resultados devem ser realizadas com precaução, uma vez que a verificação dos itens de avaliação pela população analisada foi permitida por respostas homogêneas como por exemplo, mesma universidade, curso ou país (ABDULLAH, 2005, 2006b, 2006a; BROCHADO, 2009; CALVO-PORRAL et al., 2013; LAW, 2013; MAHMOUD; KHALIFA, 2015; MANDAL; GUPTA, 2019; RODRIGUES et al., 2011; SONI; GOVENDER, 2018). Salienta-se ainda que a construção dos elementos para a investigação da qualidade de serviços em IESs varia de acordo com o tipo de instituição a ser avaliada (HAMID; YIP, 2019) uma vez que foi percebida a utilização da adaptação do SERVPERF em 9 dos 15 artigos selecionados (CALVO-PORRAl et al., 2013; DALATI; AL HAMWI, 2016; HAMID; YIP, 2019; JOHARI; ZAINAB, 2007; MAHMOUD; KHALIFA, 2015; MANDAL; GUPTA, 2019; MWIYA et al., 2019; NEJATI \& NEJATI, 2008; SONI; GOVENDEr, 2018).

Porém, de uma maneira geral, compreende-se que as cinco dimensões da qualidade de desempenho de serviços são responsáveis pela satisfação geral dos estudantes em IESs, e consequentemente, transforma-se em um relacionamento de lealdade e reputação de marketing positivo (MWIYA et al., 2019). Apesar disso, notou-se a dimensão segurança com os menores índices, representando que IESs devem colocar esforços nessas áreas (CALVOPORRAL et al., 2013; RODRIGUES et al., 2011). Por fim, observou-se a relação positiva entre a percepção da qualidade de serviços, satisfação dos estudantes, serviços e elaboração justa dos preços ofertados por instituições privadas (DALATI; AL HAMWI, 2016).

Portanto, identificou-se entre as pesquisas analisadas que ainda não há consenso na priorização das dimensões da qualidade devido às características singulares de aplicação de cada estudo. Diversos trabalhos aplicaram a ferramenta SERVPERF em instituições públicas (Tabela 2), apesar disso, como percebido por esta RSL, nenhum trabalho foi desenvolvido em IESs brasileiras, especificamente em instituições públicas. Dito isto, a próxima seção desta 
pesquisa destina-se a elaborar um questionário baseado nos itens do instrumento SERVERPF para avaliar a qualidade de serviços em um instituto federal de ensino superior.

\section{METODOLOGIA}

A partir da apresentação da problemática de pesquisa (seção 1) e dos resultados obtidos na RSL referente à aplicação do instrumento SERVPERF em IESs (seção 2), definiram

-se as próximas etapas do trabalho em: definição do ambiente de estudo (etapa 1), elaboração e aplicação do questionário piloto e final (etapa 2), análise de dados (etapa 3) e discussão dos resultados (etapa 4), descritas a seguir.

\subsection{Etapa 1: Definição do ambiente de estudo}

Definiu-se uma IP de ensino como IES a ser analisada. A instituição apresenta diversos campi no estado em que está localizada e promove a oferta de cursos de qualificação profissional, técnicos, especialização técnica de nível médio, graduação e pós graduação para mais de 36 mil alunos. Especificamente, um dos campi da instituição será abordado por promover a capacitação de estudantes do curso de bacharelado em engenharia de produção, público-alvo desta pesquisa.

Dentre os setores pedagógicos da instituição, encontra-se um departamento de suporte ao ensino (DSE). Selecionou-se esse departamento devido ao fato de fornecer suporte a todas as atividades pertinentes ao desenvolvimento do processo educativo na instituição. Atualmente, o DSE é formado por um coordenador, três servidores e duas estagiárias. As principais atribuições estão relacionadas: ao cumprimento do código de ética dos discentes, ao zelo pelo cumprimento dos horários de aula e demais atividades letivas, à confecção de documentação necessária à identificação do aluno, à atualização do registro de ocorrências diárias no sistema acadêmico e ao controle da utilização dos espaços físicos destinados às atividades de ensino no campus. Apesar de um regimento interno definido, percebeu-se que os estudantes apresentam dificuldades em compreender a oferta dos principais serviços promovidos pelo DSE. Consequentemente, dificulta-se a percepção do nível de qualidade referente aos serviços ofertados aos estudantes.

Este trabalho apresenta uma abordagem quantitativa devido à presença de cálculos numéricos promovidos por meio de técnicas estatísticas para alcançar os resultados 
(WAINER, 2007) a fim de determinar a resposta de um público-alvo (MANZATO; SANTOS, 2012). Além disso, a pesquisa possui abordagem qualitativa, pois, apesar da utilização de ferramentas estatísticas na análise de dados, os resultados obtidos foram promovidos a partir do valor, sensibilização e percepção individual dos participantes (LUDKE; ANDRÉ, 1986). Ainda, o desenvolvimento ocorreu por meio de um estudo de caso, de modo a permitir a melhora da compreensão de um evento empírico (VOSS et al., 2002; YIN, 2017) a partir da investigação aprofundada de um problema (GIL, 2007) por meio de um questionário baseado nos constructos do SERVPERF (CRONIN; TAYLOR, 1992). Este estudo de caso é caracterizado como singular, pois examina a prestação de serviços dentro de um contexto real de um único objeto de estudo (MIGUEL et al., 2012; YIN, 2001).

\subsection{Etapa 2: Elaboração e aplicação do questionário piloto e final}

A elaboração do questionário realizou-se a partir da adaptação do instrumento SERVPERF desenvolvido por Cronin e Taylor (1992). Para o estudo proposto, 10 itens foram considerados relevantes para a avaliação dos serviços do DSE (Quadro 1) para a construção de um questionário objetivo, rápido e de fácil entendimento. Os itens foram elaborados e aprovados em conjunto com o representante do setor (coordenador). A determinação dos itens deu-se a partir da realidade do departamento analisado e da necessidade de investigar oportunidades de melhorias aos serviços promovidos aos estudantes do campus.

\begin{tabular}{cl} 
& \multicolumn{1}{c}{ Quadro 1 - Itens de avaliação da qualidade dos serviços do DSE } \\
\hline $\mathbf{N}^{\mathbf{0}}$ & \multicolumn{1}{c}{ Item } \\
\hline 1 & Atualização do cadastro das salas/horários das aulas no portal acadêmico para matrículas online. \\
2 & Confeç̧ão/distribuição do documento de identificação ao aluno. \\
3 & Utilização dos laboratórios de informática. \\
4 & Atualização dos horários disponibilizados nas portas das salas/laboratórios. \\
5 & Disposição em ajudar no agendamento das provas de segunda chamada. \\
6 & Agilidade na comunicação dos acontecimentos que impactem as aulas/funcionamento do campus. \\
7 & Controle da entrada, permanência e saída de pessoas no campus. \\
8 & Cordialidade dos colaboradores. \\
9 & Horário de atendimento (7h às 22h). \\
10 & Fiscalização/aplicação de penalidades referente ao código de ética do discente no instituto. \\
\hline
\end{tabular}

Inicialmente, desenvolveu-se o questionário piloto a partir de uma reunião com o representante do DSE a fim de integrar as funções do órgão com as atividades específicas atribuidas aos alunos do curso de graduação de engenharia de produção. A coleta de dados do questionário piloto ocorreu na última semana de novembro de 2019 com 16 estudantes do 
sexto período. A avaliação da qualidade dos serviços prestados pelo DSE foi mensurada a partir de uma escala Likert cujos valores variam de 1 (muito ruim) a 5 (muito bom). De acordo com os alunos, percebeu-se que o questionário piloto apresentou perguntas concisas e objetivas. Apesar disso, sugeriu-se a inclusão de um campo extra "não sei responder" (nota zero) pois algumas dessas atividades ainda não haviam sido utilizadas pelos alunos participantes durante esta fase teste.

Após alterações realizadas, elaborou-se o questionário final. A coleta de dados para o questionário final obteve a participação de 235 alunos, dentre as quais 192 repostas são válidas, e de acordo com Hair Jr. et al. (2014), representa o mínimo de 30\% para uma população inferior a 300 participantes. Foram apenas considerados questionários respondidos por estudantes que já utilizaram algum serviço do DSE. Assim, as respostas dos itens com avaliação nula foram desconsideradas dos cálculos. A aplicação do questionário final foi realizada por meio de formulários online via Google Forms pois o período de aplicação (20 de janeiro e 20 de fevereiro de 2020) não era letivo. O fluxograma referente aos passos da elaboração do questionário é exemplificado na Figura 3.

Figura 3 - Metodologia para aplicação do questionário

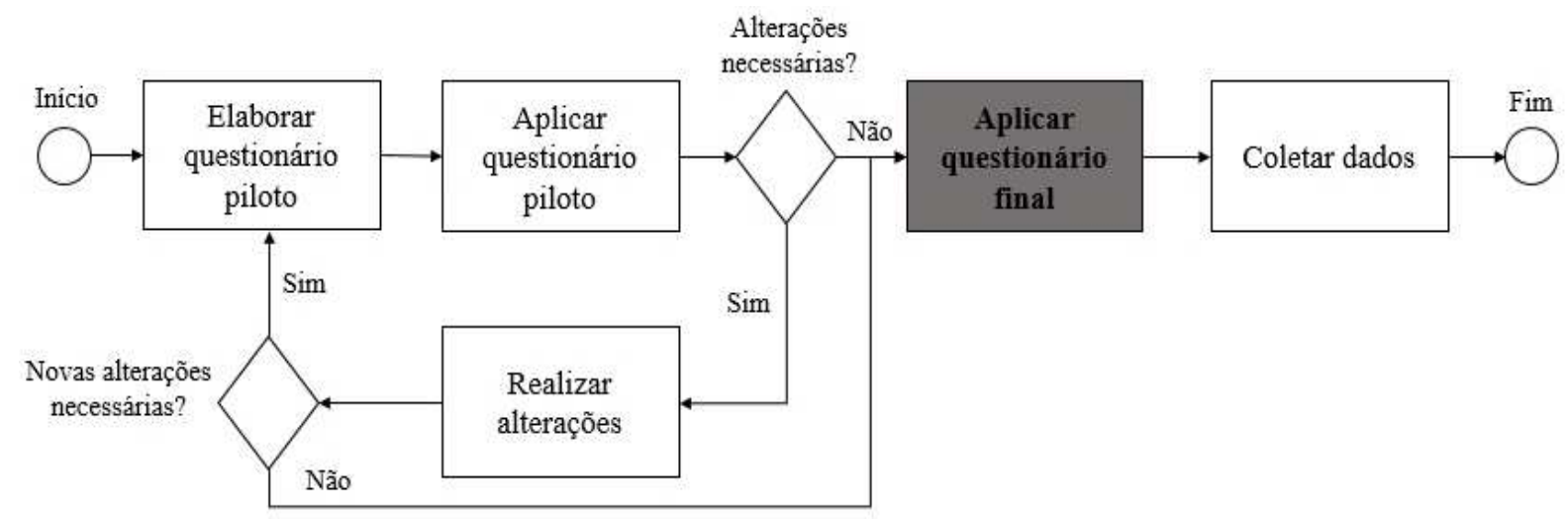

\subsection{Etapa 3: Análise de dados}

A seguir, analisaram-se os dados obtidos pela coleta dos questionários avaliados pelos alunos (seção 4.1). A análise de dados utilizou o grau de desempenho médio, calculado a partir da média aritmética do desempenho de cada item, o desvio padrão e a moda. Esta etapa utilizou a assistência do software Microsoft Excel no auxílio aos cálculos. Além disso, apresentou-se a priorização dos itens por meio da classificação dos mesmos como crítico, alto, 
moderado e baixo a partir da aplicação da análise de quartis proposta por Freitas et al. (2006) e posteriormente verificada em Freitas et al. (2009), Gonçalves; Freitas (2010) e Gonçalves; Belderrain (2012). Nesta pesquisa, objetivou-se analisar os itens de classificação crítica e alta aos serviços oferecido pelo DSE a fim de promover a reflexão imediata dos resultados, além de sugerir oportunidades de melhoria junto à equipe do setor.

\subsection{Etapa 4: Discussão dos resultados}

Por fim, a última etapa da pesquisa (seção 4.2) está destinada à discussão dos principais resultados encontrados na aplicação do questionário e confrontar com os achados da RSL desenvolvida anteriormente (seção 2). Aqui, objetiva-se identificar as contribuições (teóricas e práticas) e limitações do trabalho, auxiliando a recomendação de elaboração de pesquisas futuras.

\section{RESULTADOS E DISCUSSÕES}

\subsection{Análise de dados}

A partir da coleta de dados realizada, analisaram-se os dados por meio da média, desvio padrão e moda e classificaram-se os itens de acordo com a análise de quartis proposta por Freitas et al. (2006). Como informado anteriormente, apenas os itens caracterizados em prioridades crítica e alta serão analisados nesta pesquisa. Os itens foram classificados de acordo com as dimensões da qualidade (PARASURAMAN et al., 1988): tangibilidade (TAN), confiabilidade (CON), responsividade (RES), segurança (SEG) e empatia (EMP). O Quadro a seguir exemplifica os resultados obtidos.

\begin{tabular}{cccccc}
\multicolumn{6}{c}{ Quadro 2 - Resultados da aplicação dos questionários } \\
\hline Prioridade & Dimensão & $\mathbf{N}^{\mathbf{0}}$ & Média & Desvio Padrão & Moda \\
\hline \multirow{3}{*}{ CRÍTICA } & TAN & 7 & 2,74 & 1,29 & 2 \\
& TAN & 2 & 3,15 & 1,08 & 3 \\
& RES & 6 & 3,18 & 1,17 & 4 \\
\multirow{4}{*}{ ALTA } & RES & 4 & 3,27 & 1,12 & 4 \\
& CON & 10 & 3,28 & 1,03 & 3 \\
\multirow{4}{*}{ MODERADA } & & $\mathbf{Q 2}=\mathbf{3 , 2 8}$ & & \\
\hline & TAN & 3 & 3,32 & 1,1 & 4 \\
& CON & 1 & 3,52 & 1,03 & 4 \\
& RES & 5 & 3,65 & 0,99 & 4 \\
\hline
\end{tabular}




\begin{tabular}{llllll}
\hline \multirow{2}{*}{ BAIXA } & SEG & 8 & 4,12 & 0,92 & 4 \\
& CON & 9 & 4,54 & 0,72 & 5 \\
\hline
\end{tabular}

O controle da segurança na entrada, permanência e saída de pessoas no campus (item 7), exercida pelo DSE e estabelecida no regimento interno da instituto, envolve a equipe de vigilância, bem como os recursos empregados na segurança da instituição, uma vez que o acesso aos prédios do campus pelos estudantes é inviabilizado por não existir uma única entrada principal. Atualmente, o controle é realizado parcialmente por uma equipe de vigilantes, classificando o acesso dos motoristas ao estacionamento do campus em: alunos, servidores ou visitantes a partir da declaração verbal do motorista, sem qualquer informação de confirmação e/ou cadastramento em sistema de segurança.

Assim, sugere-se o investimento em recursos de segurança no campus a fim de facilitar o controle das pessoas dentro dos prédios, como por exemplo, a delimitação de uma portaria principal com um sistema de rastreamento por meio de catracas eletrônicas utilizando documentos de identificação (matrícula), já realizada por diversas instituições. Para a comunidade que não possui vínculo direto com a instituição, mas utiliza os seus serviços (biblioteca), sugere-se à elaboração de um cadastro temporário classificando o acesso como visitante. Além disso, estimula-se o planejamento de contratação de um sistema de videomonitoramento integrado interno (prédios) e externo (estacionamentos). Assim, gera-se melhora no controle de acesso ao campus, na segurança geral dos bens e pessoas e na integração da comunicação entre a vigilância e o DSE. De acordo com o representante do departamento, o processo relacionado à implementação de catracas eletrônicas em conjunto com o sistema de videomonitoramento já está em andamento, porém, devido à pandemia, as atividades foram paralisadas e a entrega final foi adiada para o início do segundo semestre de 2021.

Conforme informado anteriormente, a documentação do aluno está diretamente relacionada ao controle de acesso e permanência de pessoas, bem como à segurança no campus. Atualmente, a instituição fornece documento provisório em papel nos 30 primeiros dias de aula com validade de um ano, sendo gerado um novo documento provisório por ano até o final do curso. De acordo com o representante do departamento, a confecção dos documentos de identificação dos alunos (item 2) em material PVC não está sendo realizada nos últimos três anos por falta de recursos financeiros. A confecção da documentação em PVC seria compatível ao dispositivo eletrônico sugerido no monitoramento utilizado nas 
catracas eletrônicas. Ademais, o documento de identificação em formato PVC otimiza o processo de confecção, pois a elaboração ocorre apenas uma única vez durante o curso, evitando retrabalhos e atrasos na confecção.

A maioria dos alunos informou que a agilidade nas informações que se referem ao funcionamento do campus e continuidade das aulas (item 6) ainda é deficiente, causando alguns transtornos para alunos que podem receber informes tardiamente. Tais informações podem estar relacionadas a eventos extraordinários, como a impossibilidade de acessar o campus devido às inundações provocadas pelas chuvas torrenciais, ausência de água nas tubulações devido às manutenções preventivas na região, não comparecimento de algum professor para lecionar a disciplina por motivos especiais, entre outros. Atualmente, a comunicação desses eventos é realizada por meio do envio de mensagem automática via sistema acadêmico. Porém, percebe-se a ineficiência deste mecanismo devido à demora na atualização da comunicação e muitas dessas mensagens são reconhecidas como spam nos $e$ mails dos alunos. Dessa forma, recomenda-se a utilização não só de meios de comunicação oficiais da instituição, como também das ferramentas de transmissão de mensagens instantâneas por meio da integração com a equipe de tecnologia da informação, por exemplo, o envio em mala direta via SMS ou WhatsApp.

Atualmente a atualização dos horários das aulas e laboratórios de informática (item 4) é realizada manualmente a partir da fixação da informação nas portas das salas. Assim, dificulta-se o controle e monitoramento da informação, consequentemente, gerando atrasos nas correções dos horários e transtornos aos alunos e professores da instituição. Sugere-se a informatização do serviço por meio de telas disponibilizadas em pontos focais dentro dos prédios e/ou a elaboração de um sistema mobile informando as atividades e ocupação de todas as salas e laboratórios. Dessa forma, a informação torna-se ágil, confiável e atualizada em tempo real.

A fiscalização comportamental referente ao cumprimento do código de ética dentro do campus (item 10) é realizada a partir da inspeção visual, sendo apenas possível exercer esta função quando os alunos estão presencialmente nas áreas comuns do campus. Porém, o DSE não atende somente os alunos do curso de graduação em engenharia de produção, mas também outros cursos de graduação, ensino médio e cursos técnicos. Assim, sugere-se aumentar a equipe do DSE, como a contratação de mais estagiários em períodos diferentes. Por fim, em concordância com o sugerido no item 7, a fiscalização dos ambientes comuns dentro dos prédios pode ser monitorada pela própria sala do DSE, otimizando o tempo e deslocamento dos membros da equipe. De acordo com o representante do departamento, 
adquiriu-se uma televisão para videomonitoramento na sala do DSE e o projeto de instalação de 20 câmeras está em andamento.

\subsection{Discussão}

A partir da análise de quartis realizada anteriormente (seção 4.1), identificou-se que os principais itens 7,2, 6, 4 e 10 presentes nas prioridades crítica e alto, representam três dimensões da qualidade, como a: tangibilidade, responsividade e confiabilidade. Observou-se que os itens 7 e 2, apesar de abordarem a dimensão tangibilidade devido à confecção e distribuição da documentação estudantil e estrutura física para o acesso ao campus, estão diretamente relacionadas à promoção da confiabilidade dos estudantes. Esta relação é identificada devido à ineficiência do controle atual de acesso ao campus pelos estudantes pois ainda não há uma entrada principal, dificultando as atividades de monitoramento da segurança dos ambientes pelos vigilantes. Dessa firma, a revisão desses itens é importante para a promoção da confiabilidade nos serviços do DSE, como confirmado no resultado da pesquisa realizada em IESs públicas de Hamid \& Yip (2019).

Além disso, a dimensão responsividade, presente nos itens 6 e 4, demonstra que o setor apresenta uma deficiência referente à agilidade de informar possíveis alterações das atividades do campus, como: cancelamento de aulas, substituições das identificações das salas e eventos extraordinários. Apesar disso, verifica-se que tal agilidade é essencial para a melhoria da qualidade dos serviços percebida pelos estudantes em IESs (JOHARI; ZAINAB, 2007; MAHMOUD; KHALIFA, 2015). Portanto, os ajustes nesses serviços elevam as oportunidades de melhoria para a avaliação da qualidade nos serviços do DSE.

Salienta-se que os métodos para o planejamento, elaboração e desenvolvimento dos itens do questionário final foram adaptadas do instrumento de avaliação SERVPERF original a partir do entendimento das necessidades específicas do atual momento do DSE de acordo com o representante do setor no campus, assim como percebido em outras pesquisas do portfólio final na RSL (seção 2). Assim, o questionário foi desenvolvido de modo a garantir resultados satisfatórios e imediatos para a correção e melhoria da promoção apenas dos serviços prioritários pelo setor. Dessa forma, promove-se a alocação de recursos suficientes em tais itens, como confirma Abdullah (2006b).

Além dos ajustes necessários nos itens do instrumento SERVPERF à realidade do DSE, destaca-se que a importância do planejamento e utilização do teste piloto com os estudantes, assim como percebido em diversos artigos da RSL. Apesar do questionário final 
ter sido aplicado apenas por estudantes de diferentes turmas no curso de engenharia de produção, desconsiderando corpo docente e outras equipes administrativas do instituto (CALVO-PORRAL et al., 2013), influenciando as respostas dos questionários devido ao nível de experiência individual dos estudantes, esta prática permitiu aproximar os itens de avaliação ao cotidiano percebido pelos alunos da instituição. Dessa maneira, elevam-se as oportunidades de melhorias práticas nos serviços, confirmando a eficácia da ferramenta em IESs.

Esta pesquisa investigou a influência da qualidade de serviços de um setor específico de uma IP, indiretamente responsável pela promoção da educação, porém necessário ao desenvolvimento das atividades de uma instituição de ensino, assim como Johari e Zainab (2007) e Nejati e Nejati (2008) realizada em bibliotecas universitárias. Dessa forma, salientase a importância da promoção de ferramentas confiáveis e quantitativas para a melhoria contínua (BROCHADO, 2009) que também permitam a avaliação dos serviços em setores de apoio ao ensino, como o DSE, uma vez que esta atividade é necessária na maioria das organizações fornecedoras de serviços (JOHARI; ZAINAB, 2007).

De uma maneira geral, notou-se que a utilização do questionário como instrumento para avaliar a qualidade dos principais serviços ofertados pelo DSE permitiu maior proximidade entre as atribuições do setor e a percepção destas pelos alunos, principal objetivo desta pesquisa. Com isso, os benefícios da promoção da investigação da qualidade de serviços podem não ser apenas observados para o departamento no campus estudado, mas também para as outras 21 coordenadorias de apoio ao ensino no estado analisado e aos diversos setores relacionados às atividades de apoio ao ensino pedagógico dispersados nas IESs no Brasil. Desta forma, novas alterações nos constructos do instrumento devem ser realizadas de modo a garantir que os resultados sejam adequados à realidade total do ambiente investigado, indo ao encontro dos achados de Rodrigues et al. (2011) e Hamid e Yip (2019).

\section{CONSIDERAÇÕES FINAIS}

Este trabalho objetivou elaborar e aplicar um questionário com 10 itens baseado no instrumento SERVPERF para avaliar a qualidade de serviços ofertados por um órgão de apoio ao ensino em uma IES, especificamente em uma IP. Como resultados da pesquisa, identificaram-se cinco itens classificados em prioridades crítica e alta a partir da análise de quartis. Tais itens foram relacionados às dimensões da qualidade em serviços, como por exemplo a tangibilidade, responsividade e confiabilidade. Os resultados do instrumento 
permitiram que o setor compreenda quais são as principais atividades fornecidas que demandam melhorias imediatas.

Nota-se que esta pesquisa apresenta contribuições teóricas e práticas. Primeiramente, compreende-se que, até o momento de elaboração deste trabalho, para o conhecimento dos autores, ainda não foram desenvolvidas pesquisas referentes apenas à investigação do instrumento SERVPERF em IESs por meio de RSL, apesar do instrumento ter sido desenvolvido há quase três décadas. Ainda, como apresentado nos resultados da RSL, não foi verificada a investigação do SERVPERF em IP de ensino brasileiras. Dessa forma, a principal contribuição acadêmica deste trabalho está relacionada à expansão e disseminação do conhecimento desta área na literatura, como percebido na seção 2.

Além disso, percebeu-se que a aplicação do questionário na instituição analisada permitiu que o setor de apoio ao ensino seja capaz de compreender, corrigir e melhorar os serviços como prioritários pelos estudantes a fim de elevar a qualidade da promoção de suas atividades, consequentemente, promovendo a cultura de melhoria contínua no instituto. Assim, sugere-se que a repetibilidade em outras IEs no estado é viável, porém, passiva de adaptações. Ainda, esta pesquisa permitiu maior envolvimento e interação pelos estudantes em auxiliar a promoção de melhorias do órgão para o instituto.

Apesar das contribuições apresentadas, entende-se que este trabalho também apresenta limitações. Destacam-se: (i) a viabilidade para a aplicação do questionário em outras IPs no estado devem ser realizadas com cautela, uma vez que existem características singulares à este ambiente; (ii) a validação da consistência interna dos resultados da amostra a partir do alfa de Cronbach (Cronbach, 1951) não foi permitida devido à presença da atribuição da nota zero no instrumento desenvolvido, que apesar da desconsideração desses valores, impactou em resultados abaixo do aceitável; (iii) o questionário não investigou características relacionadas à dimensão empatia, considerada importante na avaliação da qualidade dos serviços; e por fim, (iv) assim como diversas pesquisas encontradas na seção 2, percebeu-se que os resultados desta pesquisa não podem ser generalizados devido à implementação do questionário em apenas um ambiente de estudo e mesmo perfil de respondentes.

Dessa maneira, fazem-se algumas sugestões para trabalhos futuros. Inicialmente, recomenda-se a realização de ajustes para permitir que o instrumento seja adequado aos diferentes setores de apoio ao ensino presentes em IESs, como por exemplo: reestruturação do questionário a partir da eliminação das notas de avaliações zero e utilização de respostas dos estudantes dos últimos dois anos do cursos de graduação para elevar a confiabilidade das respostas na amostra devido à experiência e percepção dos serviços desses estudantes, assim 
como Calvo-Porral et al. (2013), Mandal e Gupta (2019) e Mwiya et al. (2019). Além disso, deseja-se que outras pesquisas sejam realizadas a partir da investigação de diferentes públicos-alvo à setores de apoio ao ensino de modo a garantir heterogeneidade da população da amostra. Ademais, indica-se comparar os resultados da aplicação do instrumento desta pesquisa com a investigação da adaptação dos 22 itens originais do SERVPERF, independentemente dos ajustes necessários à realidade da instituição. Por fim, sugere-se a aplicação de um questionário baseado no instrumento SERVPERF voltado à promoção dos serviços de todo o ambiente da instituição, não só de um setor específico.

\section{REFERÊNCIAS}

Abdullah, F. (2005). HEdPERF versus SERVPERF: The quest for ideal measuring instrument of service quality in higher education sector. Quality Assurance in Education, 13(4), 305-328. https://doi.org/10.1108/09684880510626584.

Abdullah, F. (2006a). Measuring service quality in higher education: HEdPERF versus SERVPERF. Marketing Intelligence and Planning, 24(1), 31-47. https://doi.org/10.1108/02634500610641543

Abdullah, F. (2006b). Measuring service quality in higher education: Three instruments compared. International Journal of Research and Method in Education, 29(1), 71-89. https://doi.org/10.1080/01406720500537445

Abdullah, F. (2006c). The development of HEdPERF: A new measuring instrument of service quality for the higher education sector. International Journal of Consumer Studies, 30(6), 569-581. https://doi.org/10.1111/j.1470-6431.2005.00480.x

Acerbi, F., \& Taisch, M. (2020). A literature review on circular economy adoption in the manufacturing sector. Journal of Cleaner Production, 273, 123086. https://doi.org/10.1016/j.jclepro.2020.123086

Akdere, M., Top, M., \& Tekingündüz, S. (2020). Examining patient perceptions of service quality in Turkish hospitals: The SERVPERF model. Total Quality Management and Business Excellence, 31(3-4), 342-352. https://doi.org/10.1080/14783363.2018.1427501

Alvarenga, D. (2020). Economia - Setor de serviços cresce $1 \%$ em 2019 e tem $1^{a}$ alta em 5 anos. https://g1.globo.com/economia/noticia/2020/02/13/setor-de-servicos-cresce-1percentem-2019-diz-ibge.ghtml

Amorim, D., Batista, R., \& Neder, V. (2019). Economia \& Negócios - Com maior peso no PIB, setor de serviços puxa avanço da economia. https://economia.estadao.com.br/noticias/geral,setor-de-servicos-com-maior-peso-no-pibpuxa-avanco-da-economia,70002739128. 
Babić-Hodović, V., Arslanagić-Kalajdžić, M., Banda, A., \& Sivac, A. (2019). IPA and SERVPERF quality conceptualisations and their role in hotel services satisfaction. Tourism and Hospitality Management, 25(1), 1-17. https://doi.org/10.20867/thm.25.1.4.

Brochado, A. (2009). Comparing alternative instruments to measure service quality in higher education. Quality Assurance in Education, 17(2), 174-190. https://doi.org/10.1108/09684880910951381.

Bustos, O., \& Pomares-Quimbaya, A. (2020). Stock market movement forecast: A Systematic review. Expert Systems with Applications, 156. https://doi.org/10.1016/j.eswa.2020.113464.

Calvo-Porral, C., Lévy-Mangin, J. P., \& Novo-Corti, I. (2013). Perceived quality in higher education: An empirical study. Marketing Intelligence and Planning, 31(6), 601-619. https://doi.org/10.1108/MIP-11-2012-0136.

Coscieme, L., Mortensen, L. F., Anderson, S., Ward, J., Donohue, I., \& Sutton, P. C. (2020). Going beyond Gross Domestic Product as an indicator to bring coherence to the Sustainable Development Goals. Journal of Cleaner Production, 248. https://doi.org/10.1016/j.jclepro.2019.119232

Cronbach, L. J. (1951). Coefficient alpha and the internal structure of tests. Psychometrika, 16(3), 297-334. https://doi.org/10.1007/BF02310555

Cronin, J. J. J., \& Taylor, S. A. (1992). Measuring service quality: a re-examination and extension. Journal of Marketing, 56(3), 55-68.

Dalati, S., \& Al Hamwi, S. E. (2016). Sustainable development in higher education through service quality and price fairness: empirical evidence from private universities in Syria. $\begin{array}{lllll}\text { Entrepreneurship and } \quad \text { Sustainability } & \text { Issues, }\end{array}$ https://doi.org/10.9770/jesi.2016.4.1(3)

Devynck, F., Rousseau, A., \& Romo, L. (2019). Does repetitive negative thinking influence alcohol use? A systematic review of the literature. Frontiers in Psychology, 10(JULY). https://doi.org/10.3389/fpsyg.2019.01482

Dussault, G. (1992). A gestäo dos serviços públicos de saúde: características e exigências. Rev. Adm. Pública, 26(2), 8-19.

Dutta, S. (2019). Dimension specific technique of evaluating service quality. International Journal of Innovative Technology and Exploring Engineering, 8(12), 2109-2118. https://doi.org/10.35940/ijitee.L3289.1081219

Fitzsimmons, J. A., \& Fitzsimmons, M. J. (2014). Administração de Serviços: operações, estratégia e tecnologia da informação (7th ed.). AMGH.

Freitas, A. L. P., Manhães, N. R. C., \& Cozendey, M. I. (2006). Emprego do SERVQUAL na Avaliação da Qualidade de Serviços de Tecnologia da Informação: uma Análise Experimental. XXVI Encontro Nacional de Engenharia de Produção. 
Freitas, A. L. P., Rodrigues, S. G., \& Costa, H. G. (2009). Emprego de uma abordagem multicritério para classificação do desempenho de instituições de ensino superior. Ensaio: Avaliação e Políticas Públicas Em Educação, 17(65), 656-674. https://doi.org/10.1590/s0104-40362009000400006

Gao, W., Ding, X., Chen, R., \& Min, W. (2019). An empirical study of the role of higher education in building a green economy. Sustainability (Switzerland), 11(23), 1-14. https://doi.org/10.3390/su11236823

Gil, A. C. (2007). Métodos e Técnicas de Pesquisa Social (5th ed.). Atlas.

Glodowski, K. R., Thompson, R. H., \& Ashley Asuncion, E. (2020). Evidence-Based Recommendations for Programming Quizzes to Improve College Student Behavior in Residential Courses. Journal of Behavioral Education, 29(3), 543-570. https://doi.org/10.1007/s10864-019-09330-z

Goldstein, S. M., Johnston, R., Duffy, J., \& Rao, J. (2002). The service concept: the missing link in service design research? Journal of Operations Management, 20(2), 121-134. https://doi.org/10.1016/S0272-6963(01)00090-0

Gonçalves, T. J. M., \& Belderrain, M. C. N. (2012). Avaliação da qualidade em lan houses através da adpatação do instrumento SERVQUAL. Revista Produção Online, 12(1), 248-268. https://doi.org/https://doi.org/10.14488/1676-1901.v12i1.882

Gonçalves, T. J. M., \& Freitas, A. L. P. (2010). Emprego de um sistema web para aplicação de questionários na avaliação da qualidade em serviços. Ingepro - Inovação, Gestão e Produção, 2(1), 108-120.

Grönroos, C. (2004). Marketing: gerenciamento e serviços (Elsevier (Ed.)).

Guerrero, M., Cunningham, J. A., \& Urbano, D. (2015). Economic impact of entrepreneurial universities' activities: An exploratory study of the United Kingdom. Research Policy, 44(3), 748-764. https://doi.org/10.1016/j.respol.2014.10.008

Hair Jr., J. F., Celsi, M. W., Ortinau, D. J., \& Bush, R. P. (2014). Fundamentos de pesquisa de marketing (3rd ed.). Mc Graw-Hin.

Hamid, F. S., \& Yip, N. (2019). Comparing service quality in public vs private distance education institutions: Evidence based on Malaysia. Turkish Online Journal of Distance Education, 20(1), 17-34. https://doi.org/10.17718/tojde.522368

Hansen, D. L., Shneiderman, B., \& Smith, M. A. (2011). Analyzing social media networks with nodexl insights from a connected world. Morgan Kauffman: Burlington.

Haque, M. I., \& Sultan, Z. A. (2019). A structural equation modeling approach to validate the dimensions of SERVPERF in insurance industry of Saudi Arabia. Management Science Letters, 9(4), 495-504. https://doi.org/10.5267/j.msl.2019.1.012

IBGE. (2016). Brasil em Síntese - Instituto Brasileiro de Geografia e Estatística. https://brasilemsintese.ibge.gov.br/servicos.html 
IBGE. (2020). Produto Interno Bruto - PIB. https://www.ibge.gov.br/explica/pib.php Idrissi, N., \& Zellou, A. (2020). A systematic literature review of sparsity issues in recommender systems. Social Network Analysis and Mining, 10(1). https://doi.org/10.1007/s13278-020-0626-2

Jacob, A., Moullec, G., Lavoie, K. L., Laurin, C., Cowan, T., Tisshaw, C., Kazazian, C., Raddatz, C., \& Bacon, S. L. (2018). Supplemental Material for Impact of CognitiveBehavioral Interventions on Weight Loss and Psychological Outcomes: A Meta-Analysis. Health Psychology, 37(5), 417-432. https://doi.org/10.1037/hea0000576.supp

Johari, R., \& Zainab, A. N. (2007). Identifying what services need to be improved by measuring the library's performance. Malaysian Journal of Library and Information Science, 12(1), 35-53.

Kotler, P. (1988). Administração e Marketing (5th ed.). Altas.

Law, D. C. s. (2013). Initial assessment of two questionnaires for measuring service quality in the Hong Kong post-secondary education context. Quality Assurance in Education, 21(3), 231-246. https://doi.org/10.1108/QAE-Sep-2012-0034

Ludke, M., \& André, M. (1986). Pesquisa em educação: abordagens qualitativas. Editora Pedagógica e Universitária.

Mahmoud, A. B., \& Khalifa, B. (2015). A confirmatory factor analysis for SERVPERF instrument based on a sample of students from Syrian universities. Education and Training, 57(3), 343-359. https://doi.org/10.1108/ET-04-2014-0038

Mandal, K., \& Gupta, H. (2019). Gap versus performance based measure of pharmaceutical education service quality: An empirical comparison. Indian Journal of Pharmaceutical Education and Research, 53(3), 421-434. https://doi.org/10.5530/ijper.53.3.75

Manzato, A. J., \& Santos, A. B. (2012). A Elaboração de Questionários na Pesquisa Quantitativa. Departamento de Ciência de Computação e Estatística - IBILCE - UNESP, 117. http://www3.ufpe.br/moinhojuridico/images/ppgd/8.5a questionario_elaboracao.pdf

Martin, S., \& Grüb, B. (2020). Intensive WOM-behavior in the healthcare sector - the case of an Austrian hospital's Facebook site. International Review on Public and Nonprofit Marketing, 17(3), 331-352. https://doi.org/10.1007/s12208-020-00250-7

Miguel, P. A. C., Fleury, A., Mello, C. H. P., Nakano, D. N., Lima, E. P. de L., Turrioni, J. B., Ho, L. L., Morabito, R., Martins, R. A., Sousa, R., Costa, S. E. G., \& Pureza, V. (2012). Metodologia de Pesquisa em Engenharia de Produção e Gestão de Operações (2nd ed.). Elsevier Editora Ltda.

Ministério da Economia. (2018). Governo Federal Divulga Pesquisa Inédita Sobre Qualidade Dos Serviços Públicos. http://www.planejamento.gov.br/noticias/governo-federal-divulgapesquisa-inedita-sobre-qualidade-dos-servicos-publicos

Mohan, R., Rai, R. S., \& Banerjee, S. P. (2019). Service quality and its role in 
upselling/cross-selling. International Journal of Innovative Technology and Exploring Engineering, 9(1), 1502-1509. https://doi.org/10.35940/ijitee.A4330.119119

Moher, D., Liberati, A., Tetzlaff, J., \& Altman, D. G. (2009). Preferred reporting items for systematic reviews and meta-analyses: The PRISMA statement. PLos Medicine, 6(7), e1000097. https://doi.org/10.1371/ journal.pmed.1000097

Mwiya, B., Siachinji, B., Bwalya, J., Sikombe, S., Chawala, M., Chanda, H., Kayekesi, M., Sakala, E., Muyenga, A., \& Kaulungombe, B. (2019). Are there study mode differences in perceptions of university education service quality? Evidence from Zambia. Cogent Business and Management, 6(1), 1-19. https://doi.org/10.1080/23311975.2019.1579414

Nejati, M., \& Nejati, M. (2008). Service quality at University of Tehran Central Library. Library Management, 29(6-7), 571-582. https://doi.org/10.1108/01435120810894563

Omodero, C. O., \& Nwangwa, K. C. K. (2020). Higher education and economic growth of Nigeria: Evidence from co-integration and granger causality examination. International Journal of Higher Education, 9(3), 173-182. https://doi.org/10.5430/ijhe.v9n3p173

Pacheco, D. A. de J., Caten, C. S. ten, Jung, C. F., Navas, H. V. G., Cruz-Machado, V. A., \& Tonetto, L. M. (2019). State of the art on the role of the Theory of Inventive Problem Solving in Sustainable Product-Service Systems: Past, Present, and Future. Journal of Cleaner Production, 212, 489-504. https://doi.org/10.1016/j.jclepro.2018.11.289

Parasuraman, A., Zeithaml, V. A., \& Berry, L. L. (1985). A Conceptual Model of Service Quality and Its Implications for Future Research. Journal of Marketing, 49(4), 41. https://doi.org/10.2307/1251430

Parasuraman, A., Zeithaml, V. A., \& Berry, L. L. (1988). SERVQUAL: A multiple-item scale for measuring consumer perceptions of service quality. Journal of Retailing, 64(1), 12-40. https://doi.org/10.1016/S0148-2963(99)00084-3

Rashidi, K., Noorizadeh, A., Kannan, D., \& Cullinane, K. (2020). Applying the triple bottom line in sustainable supplier selection: A meta-review of the state-of-the-art. Journal of Cleaner Production, 269, 122001. https://doi.org/10.1016/j.jclepro.2020.122001

Rim, H., Lee, Y. A., \& Yoo, S. (2020). Polarized public opinion responding to corporate social advocacy: Social network analysis of boycotters and advocators. Public Relations Review, 46(2). https://doi.org/10.1016/j.pubrev.2019.101869

Rodrigues, L. L. R., Barkur, G., Varambally, K. V. M., \& Motlagh, F. G. (2011). Comparison of SERVQUAL and SERVPERF metrics: An empirical study. TQM Journal, 23(6), 629-643. https://doi.org/10.1108/17542731111175248

Romani, A., \& Quintino, L. (2019). Setor de serviços sustenta o crescimento do PIB de 2018. https://veja.abril.com.br/economia/setor-de-servicos-sustenta-o-crescimento-do-pib-de-2018/ Shankar, A., Datta, B., \& Jebarajakirthy, C. (2019). Are the Generic Scales Enough to Measure Service Quality of Mobile Banking? A Comparative Analysis of Generic Service Quality Measurement Scales to Mobile Banking Context. Services Marketing Quarterly, 40(3), 224-244. https://doi.org/10.1080/15332969.2019.1630176 
Shurair, A. S. A., \& Pokharel, S. (2019). Stakeholder's perception of service quality: a case in Qatar. Quality Assurance in Education, 27(4), 493-510. https://doi.org/10.1108/QAE-052017-0023

Sihag, N., \& Sangwan, K. S. (2020). A systematic literature review on machine tool energy $\begin{array}{lllll}\text { consumption. Journal of Cleaner } & 275 .\end{array}$ https://doi.org/10.1016/j.jclepro.2020.123125

Sirbu, C. (2015). Critical Analysis of Higher Education Structure, Financial Policies, Revenue Sources and Expenditures. Study Case: Romania. SSRN Electronic Journal, May. https://doi.org/10.2139/ssrn.2606230

Soni, S., \& Govender, K. (2018). The relationship between service quality dimensions and brand equity: Higher education students' perceptions. Journal of Management and Business Administration. Central Europe, 26(3), 71-87. https://doi.org/10.7206/jmba.ce.24507814.236

Stambulova, N. B., \& Wylleman, P. (2019). Psychology of athletes' dual careers: A state-ofthe-art critical review of the European discourse. Psychology of Sport and Exercise, 42(November 2018), 74-88. https://doi.org/10.1016/j.psychsport.2018.11.013

Tranfield, D., Denyer, D., \& Smart, P. (2003). Towards a Methodology for Developing Evidence-Informed Management Knowledge by Means of Systematic Review*. British Journal of Management, 14, 207-222.

Traxler, A. A., Schrack, D., \& Greiling, D. (2020). Sustainability reporting and management control - A systematic exploratory literature review. Journal of Cleaner Production, 276. https://doi.org/10.1016/j.jclepro.2020.122725

Tukker, A. (2004). Eight types of product-service system: eight ways to sustainability? Business Strategy and the Environment, 260, 246-260.

Voss, C., Tsikriktsis, N., \& Frohlich, M. (2002). Case research in operations management. International Journal of Operations \& Production Management, 22(2), 195-219. https://doi.org/10.1108/01443570210414329

Wainer, J. (2007). Métodos de pesquisa quantitativa e qualitativa para a Ciência da Computação. Atualização Em Informática, September, 1-42. http://www.pucrs.br/famat/viali/educem/material/textos/Pesquisa.pdf

Yin, R. K. (2001). Estudo de caso: planejamentos e métodos (2nd ed.). Bookman.

Yin, R. K. (2017). Case study research and applications: design and methods (Sixth edit). Sage publications.

Yu, M., \& Hyun, S. S. (2019). The impact of foreign flight attendants' service quality on behavioral intention toward their home country-applied SERVPERF model. Sustainability (Switzerland), 11(15). https://doi.org/10.3390/su11154136

Zainuddin, N., \& Gordon, R. (2020). Value creation and destruction in social marketing 
services: a review and research agenda. Journal of Services Marketing, 34(3), 347-361. https://doi.org/10.1108/JSM-01-2019-0046

\section{Como Referenciar este Artigo, conforme ABNT:}

MONTEIRO, M. B. D; HERZOG, L. G. P; CARMO, C. T; FORCELLINI. F. A. Qualidade de Serviço no Ensino Superior Público: Uma Adaptação do Servperf. Rev. FSA, Teresina, v.18, n. 01, art. 12, p. 262-288, jan. 2021.

\begin{tabular}{|l|c|c|c|c|}
\hline \multicolumn{1}{|c|}{ Contribuição dos Autores } & $\begin{array}{c}\text { M. B. D. } \\
\text { Monteiro }\end{array}$ & $\begin{array}{c}\text { L. G. P. } \\
\text { Herzog }\end{array}$ & $\begin{array}{c}\text { C. T. } \\
\text { Carmo }\end{array}$ & $\begin{array}{c}\text { F. A. } \\
\text { Forcellini }\end{array}$ \\
\hline 1) concepção e planejamento. & $\mathrm{X}$ & $\mathrm{X}$ & $\mathrm{X}$ & $\mathrm{X}$ \\
\hline 2) análise e interpretação dos dados. & $\mathrm{X}$ & $\mathrm{X}$ & $\mathrm{X}$ & $\mathrm{X}$ \\
\hline 3) elaboração do rascunho ou na revisão crítica do conteúdo. & $\mathrm{X}$ & $\mathrm{X}$ & $\mathrm{X}$ & $\mathrm{X}$ \\
\hline 4) participação na aprovação da versão final do manuscrito. & $\mathrm{X}$ & $\mathrm{X}$ & $\mathrm{X}$ \\
\hline
\end{tabular}

